\title{
EXPLICIT BOUNDS ON RETARDED GRONWALL-BELLMAN INEQUALITY
}

\author{
M. H. M. RASHID
}

\begin{abstract}
In this paper, explicit bounds on retarded Gronwall-Bellman and Bihari-like integral inequalities are established.
\end{abstract}

\section{Introduction}

The integral inequalities which provide explicit bounds on unknown functions play an important role in the development of the theory of differential and integral equations. For instance, the explicit bounds given by the well-known Gronwall ellman [2] inequality and its nonlinear generalization due to Bihari [4] are used to a considerable extent in the literature; see $[1,3,5,6,7]$ and the references cited therein. However, in certain situations the bounds provided by the above-mentioned inequalities are not directly applicable, and it is desirable to find some new estimates which will be equally important in order to achieve a diversity of desired goals. The main purpose of this paper is to establish explicit bounds on retarded Gronwall Bellman and Bihari-like inequalities which can be used to study the qualitative behavior of the solutions of certain classes of retarded differential equations.

\section{Main results}

In what follows, $\mathbb{R}$ denotes the set of real numbers; $\mathbb{R}_{+}=\left[0, \infty\left[, \mathbb{R}_{+}^{*}=\right] 0, \infty\left[, \mathbb{R}_{1}=[1, \infty[\right.\right.$, $J=[a, b]$ is the subset of $\mathbb{R}$; and ' denotes the derivative. $C\left(J, \mathbb{R}_{+}\right)$denotes the set of all continuous functions from $J$ into $\mathbb{R}_{+}$and $C^{1}(J, J)$ denotes the set of all continuously differentiable functions from $J$ into $J$.

Theorem 2.1. Let $u, g, h \in C\left(J, \mathbb{R}_{+}\right), f \in C\left(J, \mathbb{R}_{+}^{*}\right), \alpha \in C^{1}(J, J)$ be nondecreasing with $a \leq \alpha(t) \leq$ t on J. If the inequality

$$
u(t) \leq f(t)+\int_{a}^{t} g(s) u(s) d s+\int_{a}^{\alpha(t)} h(s) u(s) d s .
$$


holds, then

$$
u(t) \leq f(t) \exp [G(t)+H(t)]
$$

where

$$
\begin{gathered}
G(t)=\int_{a}^{t} g(s) d s \\
H(t)=\int_{a}^{\alpha(t)} h(s) d s .
\end{gathered}
$$

Proof. Since $f(t)$ is positive and nondecreasing we can restate (2.1) as

$$
\frac{u(t)}{f(t)} \leq 1+\int_{a}^{t} g(s) \frac{u(s)}{f(s)} d s+\int_{a}^{\alpha(t)} h(s) \frac{u(s)}{f(s)} d s .
$$

Let $r(t)=\frac{u(t)}{f(t)}$ then

$$
r(t) \leq 1+\int_{a}^{t} g(s) r(s) d s+\int_{a}^{\alpha(t)} h(s) r(s) d s .
$$

Define a function $z(t)$ by the right-hand side of (2.6) then we have

$$
z(t)=1+\int_{a}^{t} g(s) r(s) d s+\int_{a}^{\alpha(t)} h(s) r(s) d s .
$$

Then it is clear that

$$
r(t) \leq z(t), \quad z(a)=1 .
$$

Differentiate (2.7) with respect to $t$, we get

$$
z^{\prime}(t)=g(t) r(t)+h(\alpha(t)) \alpha^{\prime}(t) r(\alpha(t)) .
$$

Using (2.8), we have

$$
z^{\prime}(t) \leq g(t) z(t)+h(\alpha(t)) \alpha^{\prime}(t) z(t)
$$

Hence

$$
\frac{z^{\prime}(t)}{z(t)} \leq g(t)+h(\alpha(t)) \alpha^{\prime}(t)
$$

So

$$
z(t) \leq \exp [G(t)+H(t)],
$$

where $G(t)$ and $H(t)$ are defined by (2.3) and (2.4). Therefore

$$
r(t) \leq \exp [G(t)+H(t)]
$$

Hence

$$
u(t) \leq f(t) \exp [G(t)+H(t)]
$$


Theorem 2.2. Let $u, g, h \in C\left(J, \mathbb{R}_{+}\right), f \in C\left(J, \mathbb{R}_{+}^{*}\right), \alpha \in C^{1}(J, J)$ be nondecreasing with $a \leq \alpha(t) \leq$ $t$ on $J$ and $p>1$ is a constant. If the inequality

$$
u^{p}(t) \leq f^{p}(t)+\int_{a}^{t} g(s) u(s) d s+\int_{a}^{\alpha(t)} h(s) u(s) d s .
$$

holds, then

$$
u(t) \leq f(t)\left[1+\left(\frac{p-1}{p}\right)[Q(t)+W(t)]\right]^{\frac{1}{p-1}},
$$

where

$$
Q(t)=\int_{a}^{t} f^{1-p}(s) g(s) d s
$$

and

$$
W(t)=\int_{a}^{t} f^{1-p}(s) h(s) d s .
$$

Proof. Since $f(t)$ is positive and nondecreasing we can rewrite (2.15) as

$$
\frac{u^{p}(t)}{f^{p}(t)} \leq 1+\int_{a}^{t} g(s) f^{1-p}(s) \frac{u(s)}{f(s)} d s+\int_{a}^{\alpha(t)} h(s) f^{1-p}(s) \frac{u(s)}{f(s)} d s .
$$

Let $r(t)=\frac{u(t)}{f(t)}$, then

$$
r^{p}(t) \leq 1+\int_{a}^{t} g(s) f^{1-p}(s) r(s) d s+\int_{a}^{\alpha(t)} h(s) f^{1-p}(s) r(s) d s
$$

Define a function $z(t)$ by the right-hand side of (2.20) then we have

$$
z(t)=1+\int_{a}^{t} g(s) f^{1-p}(s) r(s) d s+\int_{a}^{\alpha(t)} h(s) f^{1-p}(s) r(s) d s .
$$

Then it is clear that

$$
r^{p}(t) \leq z(t), z(a)=1
$$

Differentiate (2.21) with respect to $t$, we have

$$
\begin{aligned}
z^{\prime}(t) & =g(t) f^{1-p}(t) r(t)+h(\alpha(t)) f^{1-p}(\alpha(t)) \alpha^{\prime}(t) r(\alpha(t)) \\
& \leq g(t) f^{1-p}(t) z^{\frac{1}{p}}(t)+h(\alpha(t)) f^{1-p}(\alpha(t)) \alpha^{\prime}(t) z^{\frac{1}{p}}(t) .
\end{aligned}
$$

Hence

$$
z^{\frac{-1}{p}}(t) z^{\prime}(t) \leq g(t) f^{1-p}(t)+h(\alpha(t)) f^{1-p}(\alpha(t)) \alpha^{\prime}(t) .
$$

Or

$$
\frac{d z(t)}{z^{\frac{1}{p}}(t)} \leq\left(g(t) f^{1-p}(t)+h(\alpha(t)) f^{1-p}(\alpha(t)) \alpha^{\prime}(t)\right) d t .
$$


Integrating From $a$ to $t$, and making change of variable, we have

$$
\frac{p}{p-1} z^{\frac{p-1}{p}}(t)-\frac{p}{p-1} \leq Q(t)+W(t)+C .
$$

Using $z(a)=1$, we have $C \geq 0$. Hence

$$
z(t) \leq\left[1+\left(\frac{p-1}{p}\right)[Q(t)+W(t)]\right]^{\frac{p}{p-1}},
$$

where $Q(t)$ and $W(t)$ are defined by (2.17) and (2.18). Therefore,

$$
r(t) \leq\left[1+\left(\frac{p-1}{p}\right)[Q(t)+W(t)]\right]^{\frac{1}{p-1}} .
$$

So

$$
u(t) \leq f(t)\left[1+\left(\frac{p-1}{p}\right)[Q(t)+W(t)]\right]^{\frac{1}{p-1}}
$$

Theorem 2.3. Let $u, g, h \in C\left(J, \mathbb{R}_{+}\right), f \in C\left(J, \mathbb{R}_{+}^{*}\right)$ be nondecreasing and $p>1$ is a constant. Suppose that $\alpha \in C^{1}(J, J)$ nondecreasing with $a \leq \alpha(t) \leq t$ on $J$. If the inequality

$$
u(t) \leq f(t)+\int_{a}^{t} g(s) u(s) d s+\int_{a}^{\alpha(t)} h(s) u^{p}(s) d s
$$

holds then

$$
u(t) \leq f(t) z(t)
$$

where

$$
\begin{gathered}
z(t)=[w(t)]^{\frac{1}{1-p}} \\
w(t) \leq e^{(1-p) A(t)} \times\left\{\int_{a}^{t} e^{(p-1) A(s)}\left[h(\alpha(s)) f^{p-1}(\alpha(s)) \alpha^{\prime}(s)\right] d s+1\right\}
\end{gathered}
$$

and

$$
A(t)=\int_{a}^{t} g(s) d s
$$

Proof. Since $f(t)$ is positive and nondecreasing we can rewrite (2.29) as

$$
\frac{u(t)}{f(t)} \leq f(t)+\int_{a}^{t} g(s) \frac{u(s)}{f(s)} d s+\int_{a}^{\alpha(t)} h(s) f^{1-p}(s) \frac{u^{p}(s)}{f^{p}(s)} d s .
$$

If we set $r(t)=\frac{u(t)}{f(t)}$, then

$$
r(t) \leq 1+\int_{a}^{t} g(s) r(s) d s+\int_{a}^{\alpha(t)} h(s) f^{1-p}(s) r^{p}(s) d s .
$$


Define a function $z(t)$ by the right-hand side of (2.35) then we have

$$
z(t)=1+\int_{a}^{t} g(s) r(s) d s+\int_{a}^{\alpha(t)} h(s) f^{1-p}(s) r^{p}(s) d s .
$$

Then it is clear that

$$
r(t) \leq z(t), \quad z(a)=1 .
$$

Differentiate both sides of (2.36) with respect to $t$ we get

$$
\begin{aligned}
z^{\prime}(t) & =g(t) r(t)+h(\alpha(t)) f^{p-1}(\alpha(t)) \alpha^{\prime}(t) r^{p}(\alpha(t)) \\
& \leq g(t) z(t)+h(\alpha(t)) f^{p-1}(\alpha(t)) \alpha^{\prime}(t) z^{p}(t) .
\end{aligned}
$$

Therefore,

$$
z^{\prime}(t)-g(t) z(t) \leq h(\alpha(t)) f^{p-1}(\alpha(t)) \alpha^{\prime}(t) z^{p}(t) .
$$

Let $w(t)=z^{1-p}(t)$ then $w^{\prime}(t)=(1-p) z^{-p}(t) z^{\prime}(t)$. Hence

$$
\begin{gathered}
z^{\prime}(t)=\frac{1}{1-p} w^{\prime}(t) z^{p}(t) . \\
z(t)=w(t) z^{p}(t) .
\end{gathered}
$$

Substitute (2.39) \& (2.40) into (2.38) we have

$$
\frac{1}{1-p} w^{\prime}(t)-g(t) w(t) \leq h(\alpha(t)) f^{p-1}(\alpha(t)) \alpha^{\prime}(t) .
$$

Therefore

$$
\left[e^{(p-1) A(t)} w(t)\right]^{\prime} \leq e^{(p-1) A(t)} \times\left[(p-1) h(\alpha(t)) f^{p-1}(\alpha(t)) \alpha^{\prime}(t)\right] .
$$

Integrating from $a$ to $t$ we have

$$
w(t) \leq e^{(1-p) A(t)} \times\left\{\int_{a}^{t} e^{(p-1) A(s)}\left[h(\alpha(s)) f^{p-1}(\alpha(s)) \alpha^{\prime}(s)\right] d s+C\right\} .
$$

But $w(a)=z(a)=1$ so we have $C \geq 1$. Therefore

$$
z(t)=[w(t)]^{\frac{1}{1-p}} .
$$

Hence

$$
u(t) \leq f(t) z(t)
$$

Theorem 2.4. Let $u, g, h \in C\left(J, \mathbb{R}_{+}\right), f \in C\left(J, \mathbb{R}_{+}^{*}\right)$ and $\alpha \in C^{1}(J, J)$ be nondecreasing with $a \leq$ $\alpha(t) \leq t$ on $J$. For $i=1,2$, let $\psi_{i} \in C\left(\mathbb{R}_{+}, \mathbb{R}_{+}\right)$be nondecreasing functions with $\psi_{i}(u)>0$ for $u>0$ and $\frac{\psi_{i}(u(t))}{f(t)} \leq \psi_{i}\left(\frac{u(t)}{f(t)}\right)$. If the inequality

$$
u(t) \leq f(t)+\int_{a}^{t} g(s) \psi_{1}(u(s)) d s+\int_{a}^{\alpha(t)} h(s) \psi_{2}(u(s)) d s .
$$

Then for $a \leq t \leq t_{1}$, 
(i) in case $\psi_{1}(u) \leq \psi_{2}(u)$,

$$
u(t) \leq f(t) \Psi_{2}^{-1}\left[\Psi_{2}(1)+G(t)+H(t)\right] .
$$

(ii) in case $\psi_{2}(u) \leq \psi_{1}(u)$,

$$
u(t) \leq f(t) \Psi_{1}^{-1}\left[\Psi_{1}(1)+G(t)+H(t)\right],
$$

where $G(t)$ and $H(t)$ are defined by (2.3) and (2.4) and for $i=1,2, \Psi_{i}^{-1}$ are the inverse functions of

$$
\Psi_{i}(\mu)=\int_{\mu_{0}}^{\mu} \frac{d s}{\psi_{i}(s)}, \quad \mu>0, \mu_{0}>0,
$$

and $t_{1} \in J$ is chosen so that

$$
\Psi_{i}(1)+G(t)+H(t) \in \operatorname{Dom}\left(\Psi_{i}^{-1}\right)
$$

respectively, for all $t$ lying in the interval $\left[a, t_{1}\right]$.

Proof. Since $f(t)$ is positive and nondecreasing we can be restate (2.45) as

$$
\begin{aligned}
\frac{u(t)}{f(t)} & \leq 1+\int_{a}^{t} g(s) \frac{\psi_{1}(u(s))}{f(s)} d s+\int_{a}^{\alpha(t)} h(s) \frac{\psi_{2}(u(s))}{f(s)} d s \\
& \leq 1+\int_{a}^{t} g(s) \psi_{1}\left(\frac{u(s)}{f(s)}\right) d s+\int_{a}^{\alpha(t)} h(s) \psi_{2}\left(\frac{u(s)}{f(s)}\right) d s .
\end{aligned}
$$

Let $r(t)=\frac{u(t)}{f(t)}$. Hence we have

$$
r(t) \leq 1+\int_{a}^{t} g(s) \psi_{1}(r(s)) d s+\int_{a}^{\alpha(t)} h(s) \psi_{2}(r(s)) d s .
$$

Define $z(t)$ by the right-hand side of (2.49) we have

$$
z(t)=1+\int_{a}^{t} g(s) \psi_{1}(r(s)) d s+\int_{a}^{\alpha(t)} h(s) \psi_{2}(r(s)) d s .
$$

Then it is clear that

$$
r(t) \leq z(t), \quad z(a)=1 .
$$

Now

$$
\begin{aligned}
z^{\prime}(t) & =g(t) \psi_{1}(r(t))+h(\alpha(t)) \alpha^{\prime}(t) \psi_{2}(r(\alpha(t))) \\
& \leq g(t) \psi_{1}(z(t))+h(\alpha(t)) \alpha^{\prime}(t) \psi_{2}(z(t)) .
\end{aligned}
$$

In case $\psi_{1}(r(t)) \leq \psi_{2}(r(t))$ we have

$$
z^{\prime}(t) \leq \psi_{2}(z(t))\left[g(t)+h(\alpha(t)) \alpha^{\prime}(t)\right] .
$$


Therefore,

$$
\frac{d}{d t} \Psi_{2}(z(t))=\frac{z^{\prime}(t)}{\psi_{2}(z(t))}=\left[g(t)+h(\alpha(t)) \alpha^{\prime}(t)\right] .
$$

Integrating (2.53) from $a$ to $t$ and using the condition $z(a)=1$ we get

$$
\Psi_{2}(z(t))=G(t)+H(t)+\Psi_{2}(1),
$$

where $G(t)$ and $H(t)$ are defined by (2.3) and (2.4). Therefore

$$
\Psi_{2}(z(t))=G(t)+H(t)+\Psi_{2}(1) .
$$

Hence

$$
z(t)=\Psi_{2}^{-1}\left[G(t)+H(t)+\Psi_{2}(1)\right] .
$$

Using (2.51) we have the desired result. Since the proof of case (ii) is similar we omit the details.

Theorem 2.5. Let $u, g, h \in C\left(J, \mathbb{R}_{+}\right), f \in C\left(J, \mathbb{R}_{+}^{*}\right) \alpha \in C^{1}(J, J)$ be nondecreasing with $a \leq \alpha(t) \leq t$ on $J$ and $p>1$ is a constant. For $i=1,2$, Let $\psi_{i} \in C\left(\mathbb{R}_{+}, \mathbb{R}_{+}\right)$be nondecreasing functions with $\psi_{i}(u)>0$ for $u>0$ and $\frac{\psi_{i}(u(t))}{f(t)} \leq \psi_{i}\left(\frac{u(t)}{f(t)}\right)$. If the inequality

$$
u^{p}(t) \leq f^{p}(t)+\int_{a}^{t} g(s) \psi_{1}(u(s)) d s+\int_{a}^{\alpha(t)} h(s) \psi_{2}(u(s)) d s .
$$

then for $a \leq t \leq t_{1}$,

(i) in case $\psi_{1}(u) \leq \psi_{2}(u)$,

$$
u(t) \leq f(t)\left[\Psi_{2}^{-1}\left[\Psi_{2}(1)+M(t)+L(t)\right]\right]^{\frac{1}{p}} .
$$

(ii) in case $\psi_{2}(u) \leq \psi_{1}(u)$,

$$
u(t) \leq f(t)\left[\Psi_{1}^{-1}\left[\Psi_{1}(1)+M(t)+L(t)\right]\right]^{\frac{1}{p}} .
$$

Where $M(t)$ and $L(t)$ are defined by

$$
\begin{gathered}
M(t)=\int_{a}^{t} f^{1-p}(s) g(s) d s, \\
L(t)=\int_{a}^{\alpha(t)} f^{1-p}(s) h(s) d s .
\end{gathered}
$$

and for $i=1,2, \Psi_{i}^{-1}$ are the inverse functions of

$$
\Psi_{i}(\mu)=\int_{\mu_{0}}^{\mu} \frac{d s}{\psi_{i}\left(s^{\frac{1}{p}}\right)}, \quad \mu>0, \mu_{0}>0
$$

and $t_{2} \in J$ is chosen so that

$$
\Psi_{i}(1)+G(t)+H(t) \in \operatorname{Dom}\left(\Psi_{i}^{-1}\right),
$$

respectively, for all $t$ lying in the interval $\left[a, t_{2}\right]$. 
Proof. Since $f(t)$ is positive and nondecreasing we can be restate (2.57) as

$$
\begin{aligned}
\frac{u^{p}(t)}{f^{p}(t)} & \leq 1+\int_{a}^{t} g(s) f^{1-p}(s) \frac{\psi_{1}(u(s))}{f(s)} d s+\int_{a}^{\alpha(t)} h(s) f^{1-p}(s) \frac{\psi_{2}(u(s))}{f(s)} d s \\
& \leq 1+\int_{a}^{t} g(s) f^{1-p}(s) \psi_{1}\left(\frac{u(s)}{f(s)}\right) d s+\int_{a}^{\alpha(t)} h(s) f^{1-p}(s) \psi_{2}\left(\frac{u(s)}{f(s)}\right) d s .
\end{aligned}
$$

Let $r(t)=\frac{u(t)}{f(t)}$ we have

$$
r^{p}(t) \leq 1+\int_{a}^{t} g(s) f^{1-p}(s) \psi_{1}(r(s)) d s+\int_{a}^{\alpha(t)} h(s) f^{1-p}(s) \psi_{2}(r(s)) d s .
$$

Define $z(t)$ by the right-hand side of (2.63) we have

$$
z(t)=1+\int_{a}^{t} g(s) f^{1-p}(s) \psi_{1}(r(s)) d s+\int_{a}^{\alpha(t)} h(s) f^{1-p}(s) \psi_{2}(r(s)) d s .
$$

Then it is clear that

$$
r(t) \leq z^{\frac{1}{p}}(t), \quad z(a)=1 .
$$

Differentiate (2.64) we get

$$
\begin{aligned}
z^{\prime}(t) & =g(t) f^{1-p}(t) \psi_{1}(r(t))+h(\alpha(t)) f^{1-p}(\alpha(t)) \alpha^{\prime}(t) \psi_{2}(r(\alpha(t))) d s \\
& \leq g(t) f^{1-p}(t) \psi_{1}\left(z^{\frac{1}{p}}(t)\right)+h(\alpha(t)) f^{1-p}(\alpha(t)) \alpha^{\prime}(t) \psi_{2}\left(z^{\frac{1}{p}}(t)\right) .
\end{aligned}
$$

Now if $\psi_{1}(z(t)) \leq \psi_{2}(z(t))$ we have

$$
z^{\prime}(t) \leq \psi_{2}\left(z^{\frac{1}{p}}(t)\right)\left[g(t) f^{1-p}(t)+h(\alpha(t)) f^{1-p}(\alpha(t)) \alpha^{\prime}(t)\right] .
$$

Hence

$$
\Psi_{2}(z(t)) \leq\left[\Psi_{2}(1)+M(t)+L(t)\right]
$$

where $M(t), L(t), \Psi_{2}$ are defined by (2.60), (2.61) and (2.62), respectively. Therefore

$$
z(t) \leq \Psi_{2}^{-1}\left[\Psi_{2}(1)+M(t)+L(t)\right]
$$

Hence

$$
u(t) \leq f(t)\left[\Psi_{2}^{-1}\left[\Psi_{2}(1)+M(t)+L(t)\right]\right]^{\frac{1}{p}}
$$

Since the proof of case (ii) is similar we omit the details. 
Theorem 2.6. Let $u \in C\left(J, \mathbb{R}_{1}\right), f \in C\left(J, \mathbb{R}_{+}^{*}\right), g, h \in C\left(J, \mathbb{R}_{+}\right)$and $\alpha \in C^{1}(J, J)$ be nondecreasing with $a \leq \alpha(t) \leq t$ for all $t \in J$. If the inequality

$$
u(t) \leq f(t)+\int_{a}^{t} g(s) u(s) \ln (u(s)) d s+\int_{a}^{\alpha(t)} h(s) u(s) \ln (u(s)) d s
$$

holds then

$$
u(t) \leq f(t)\left[1-\frac{1}{2}(X(t)+Y(t))\right]^{2},
$$

where

$$
\begin{gathered}
X(t)=\int_{a}^{t} g(s) f^{\frac{1}{2}}(s) d s, \\
Y(t)=\int_{a}^{\alpha(t)} g(s) f^{\frac{1}{2}}(s) d s .
\end{gathered}
$$

Proof. Use the fact that $\ln (x)<\sqrt{x}$ for all $x \in(1, \infty)$. Then (2.66) can be restate as

$$
u(t) \leq f(t)+\int_{a}^{t} g(s) u^{\frac{3}{2}}(s) d s+\int_{a}^{\alpha(t)} h(s) u^{\frac{3}{2}}(s) d s .
$$

Since $f(t)$ is positive and nondecreasing function, we can be restate (2.70) as

$$
\frac{u(t)}{f(t)} \leq 1+\int_{a}^{t} g(s) f^{\frac{1}{2}}(s) \frac{u^{\frac{3}{2}}(s)}{f^{\frac{3}{2}}(s)} d s+\int_{a}^{\alpha(t)} h(s) f^{\frac{1}{2}}(s) \frac{u^{\frac{3}{2}}(s)}{f^{\frac{3}{2}}(s)} d s .
$$

Let $r(t)=\frac{u(t)}{f(t)}$ then (2.71) can be restate as

$$
r(t) \leq 1+\int_{a}^{t} g(s) f^{\frac{1}{2}}(s) r^{\frac{3}{2}}(s) d s+\int_{a}^{\alpha(t)} h(s) f^{\frac{1}{2}}(s) r^{\frac{3}{2}}(s) d s .
$$

Define a function $z(t)$ by the right-hand side of (2.72), then we have

$$
z(t)=1+\int_{a}^{t} f^{\frac{1}{2}}(s) g(s) r^{\frac{3}{2}}(s) d s+\int_{a}^{\alpha(t)} h(s) f^{\frac{1}{2}}(s) r^{\frac{3}{2}}(s) d s .
$$

Then it is clear that

$$
r(t) \leq z(t), \quad z(a)=1 .
$$

Differentiate (2.73) with respect to $t$, we have

$$
\begin{aligned}
z^{\prime}(t) & =f^{\frac{1}{2}}(t) g(t) r^{\frac{3}{2}}(t)+h(\alpha(t)) f^{\frac{1}{2}}(\alpha(t)) r^{\frac{3}{2}}(\alpha(t)) \alpha^{\prime}(t) \\
& \leq f^{\frac{1}{2}}(t) g(t) z^{\frac{3}{2}}(t)+h(\alpha(t)) f^{\frac{1}{2}}(\alpha(t)) z^{\frac{3}{2}}(\alpha(t)) \alpha^{\prime}(t) \\
& \leq f^{\frac{1}{2}}(t) g(t) z^{\frac{3}{2}}(t)+h(\alpha(t)) f^{\frac{1}{2}}(\alpha(t)) z^{\frac{3}{2}}(t) \alpha^{\prime}(t)
\end{aligned}
$$

Hence

$$
z^{\frac{-3}{2}}(t) z^{\prime}(t) \leq f^{\frac{1}{2}}(t) g(t)+h(\alpha(t)) f^{\frac{1}{2}}(\alpha(t)) \alpha^{\prime}(t) .
$$


Or

$$
\frac{d z(t)}{z^{\frac{3}{2}}(t)} \leq f^{\frac{1}{2}}(t) g(t)+h(\alpha(t)) f^{\frac{1}{2}}(\alpha(t)) \alpha^{\prime}(t) .
$$

Integrating from $a$ to $t$ and making change of variable, we have

$$
-2 z^{\frac{-1}{2}}(t)+2 \leq[X(t)+Y(t)+C] .
$$

where $X(t), Y(t)$ are defined by (2.68) and (2.69) and $C$ is a constant. using the condition (2.74), we have $C \geq 0$. Then

$$
z(t) \leq\left[1-\frac{1}{2}(X(t)+Y(t))\right]^{2}
$$

Therefore,

$$
u(t) \leq f(t)\left[1-\frac{1}{2}(X(t)+Y(t))\right]^{2}
$$

\section{Acknowledgement}

The author thank the referee for useful comments and suggestions that will improve the quality of this paper.

\section{References}

[1] R. P. Agarwal, E. Thandapani, Remarks on generalizations of Gronwall inequality, Chinese J. Math., 9:2 (1981), $1-22$.

[2] R. Bellman, The stability of solutions of linear differential equations, Duke Math. J , 10 (1943), $643-647$.

[3] R. Bellman, The boundedness of solutions of linear differential equations, Duke Math. J, 14 (1943), 83-97.

[4] I. Bihari, A generalization of lemma of Bellman and its application to uniqueness problem of diĂerential equations, Acta Math. Sci. Hungar., 7 (1965), 81-94.

[5] I. Bihari, The asymptotic behaviour of a system of non-linear diÄerential equations, Publ. Math. Inst. Hung. Acad. Sci., 8 (1964), 475-488.

[6] B. G. Pachpatte, On some generalizations of Bellman's lemma, J. Math. Anal. Appl., 5 (1975), 141-150.

[7] B. G. Pachpatte, Explicit bounds on certain integral inequalities, J. Math. Anal. Appl., 267(2002), $48-61$.

Department of Mathematics \& Statistics, Faculty of Science P.O.Box(7), Mu'tah university, Mu'tah-Jordan. E-mail: malik_okasha@yahoo.com 